\title{
Endocrine Pathology Society Hubert Wolfe Award for 2019: Call for Nominations
}

Published online: 26 November 2019

(C) Springer Science+Business Media, LLC, part of Springer Nature 2019
The Endocrine Pathology Society invites nominations for the Hubert Wolfe Award for 2019. This award, established in memory of Dr. Hubert Wolfe, is awarded annually at the discretion of the Endocrine Pathology Society to honor an outstanding original research paper in the field of endocrine pathology published during the previous calendar year by a pathologist in training. The award and accompanying prize will be awarded at the Society's annual meeting in March 2020.

Nominations should be sent to the Wolfe award committee:

Ronald A. DeLellis, M.D.

Lifespan Academic Medical Center

Email: rdelellis@lifespan.org
Arthur S. Tischler, M.D.

Tufts Medical Center

Email: atischler@tuftsmedicalcenter.org

Rony Ghossein

Memorial Sloan Kettering Cancer Center

Email: ghosseir@MSKCC.ORG

For additional information, please visit the Endocrine Pathology Society website at www.endocrine-pathology.org.

Publisher's Note Springer Nature remains neutral with regard to jurisdictional claims in published maps and institutional affiliations. 